Résumés des conférences et travaux

\title{
Épigraphie romaine et histoire sociale du monde romain
}

Ségolène Demougin

\section{(2) OpenEdition}

Journals

Édition électronique

URL : https://journals.openedition.org/ashp/229

DOI : 10.4000/ashp.229

ISSN : 1969-6310

Éditeur

Publications de l'École Pratique des Hautes Études

Édition imprimée

Date de publication : 1 octobre 2008

Pagination : 109-111

ISSN : 0766-0677

\section{Référence électronique}

Ségolène Demougin, «Épigraphie romaine et histoire sociale du monde romain », Annuaire de l'École pratique des hautes études (EPHE), Section des sciences historiques et philologiques [En ligne], 139 | 2008, mis en ligne le 04 décembre 2008, consulté le 12 juillet 2021. URL : http://journals.openedition.org/ ashp/229; DOI : https://doi.org/10.4000/ashp.229 


\title{
ÉPIGRAPHIE ROMAINE ET HISTOIRE SOCIALE DU MONDE ROMAIN
}

\author{
Directeur d'études : $\mathrm{M}^{\mathrm{me}}$ Ségolène Demougin
}

Programme de l'année 2006-2007 : I. Armée et société : à propos des officiers équestres. II. Documents épigraphiques du Irr s. av. J.-C. au IV s. apr. J.-C. (suite).

Les travaux de l'année académique ont été essentiellement consacrés aux nouveaux documents publiés et aux textes déjà connus et réinterprétés. Comme d'habitude, on répartira la matière traitée en plusieurs grands chapitres. On rappellera aussi que l'Année épigraphique, 2003 est parue en octobre 2006.

En ce qui concerne l'ordre équestre, le directeur d'études, a présenté un texte nouveau bilingue de Luna, publié par G. Menella, Epigraphica, 68 (2006), p. 414 et s., mentionnant un certain Titinius praefectus fabrum, honoré par un commune (correspondant au grec koinon) inconnu; on a d'abord analysé les occurrences, relativement rares du mot dans l'épigraphie latine. L'établissement de la généalogie de la famille, connue de la fin de la République jusqu'au II $^{\mathrm{e}}$ s. apr. J.-C., a été fait, mettant en évidence les liens de cette gens avec la famille impériale, surtout Claude et Néron, règne sous lequel elle connut son acmè, en la personne de L. Titinius Glaucus Lucretianus. Celui-ci ayant honoré, en même temps que Néron, Poppée, le directeur d'études, recherchant les hommages à la seconde femme de Néron, a soumis à son auditoire une nouvelle inscription de Piazza Armerina en Sicile, qui a échappé à l'attention de l'Année épigraphique, où l'épouse de l'empereur est honorée par un certain Honoratus (G. Manganaro, Paläste und Hütte, Berlin, 1979 [Mayence, 1982], p. 498).

Passant au début du $\mathrm{III}^{\mathrm{e}} \mathrm{s}$., on a présenté à nouveau la personnalité d'Aurelius Septimius Heraclitus, mentionné sans indication de rang ou de fonction, dans une inscription de Castel Porziano, près de Rome, récemment publiée ( $A E, 2003$, p. 286); il devait finir sa carrière comme préfet d'Égypte en 215, avec un avancement rapide qui ne lui porta pas chance. De la même aire chronologique, on a signalé (I. Perge, 283) un nouveau cursus, celui de de Ti. Claudius Plotinus, dont certaines fonctions demandent encore des éclaircissementts, comme celle de procurator Narbonensis et (orae maritimae?) Liguriae, la suite étant plus classique, avec les procuratèles du diocèse de Leptis, de la Pamphylie et Lycie, puis de la Lycie. Le mot $\dot{\alpha} \pi \circ \delta \varepsilon \delta \varepsilon 1 \gamma \mu \varepsilon ́ v o \varsigma$, en latin designatus, un hapax dans ce contexte, qui termine la carrière reste mystérieux : pour l'éditeur, il atteste d'une admission dans le sénat et d'une élection à une magistrature non mentionnée et qui, en l'espèce, ne pourrait être que la préture; mais on pourrait aussi penser à d'autres solutions, et se demander, par exemple si le terme n'est pas employé, à contre-sens, pour indiquer la promotion d'un procurateur, effectuée lors du « mouvement». Pour cette procédure, on se reportera aux Lettres de Fronton à Marc Aurèle César (V, 37 v. d. H.). On a aussi insisté sur la mention du père de Plotinus,

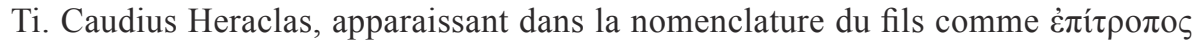




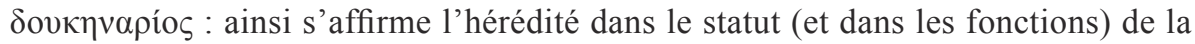
dignité équestre, qui originellement était personnelle et viagère, et qui devient de plus en plus familiale et héréditaire.

Ayant eu communication dès sa sortie du recueil des inscriptions de Kaunos, le directeur d'études est revenu sur le cursus de Ti. Claudius Clemens I. Kaunos, 124 (SEG, 44 [1994], $891=A E, 1994,1719)$, où apparaissent enfin, après la préfecture des ouvriers, les étapes du long service militaire du procurateur, et deux procuratèles, considérée de rang sexagénaire, le gouvernement de la Corse, l'autre ducénaire, la fonction d'idiologue. On connaissait déjà (cf. H.-G. Pflaum, $C P, 48)$ un procurateur de Corse homonyme en fonction entre 77 et 79 , assimilé à un préfet de la flotte d'Alexandrie connu par un diplôme militaire de 86 . Cette identification pose problème : en effet, le

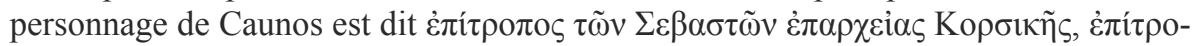

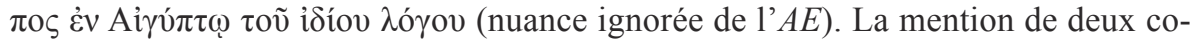
régents et celle de la préfecture des ouvriers nous contraindraient à proposer une datation dans la seconde moitié du $\mathrm{II}^{\mathrm{e}} \mathrm{s}$., et donc à dissocier les deux homonymes, qui ont exercé les mêmes fonctions à près d'un siècle d'intervalle. Mais il est préférable de considérer que les deux Augustes mentionnés se sont succédé. Dans ce cas, on pourrait envisager que Claudius Clemens, en fonctions sous Vespasien et Titus, ait poursuivi son gouvernement au début du règne de Domitien, thèse en faveur de l'asssimilation. Ajoutons que nos sources sont à peu près muettes sur l'administration de la Corse après 125. Enfin, la présentation de la publication des inscriptions de Tyr, due à J.-P. Rey Coquais, a donné l'occasion de revenir sur les quelques procurateurs figurant dans la documentation, tout en laissant de côté le très célèbre jurisconsulte Ulpien, préfet du prétoire assassiné en 228; on a donc insisté sur Ti. Iulius Alexander, procurateur de Néron et patron de la cité, et Saluius Theodorus, u(ir) e(gregius), praes(es) prouin(iae) Phoenices, sous Claude le Gothique (268-270). À propos de celui-ci, le directeur d'études est revenu sur les Saluii d'extraction équestre, en éliminant de cette aristocratie le Saluius connu à Alba Pompeia (ZPE, 40 [1980], p. 253 [AE, 1980, 495] $=S I, 17$, p. 76).

Le directeur d'études est revenu longuement sur le problème épineux de la fonction du pro legato, qu'il faut entendre de manière différente : au début de l'Empire, elle est confiée à un jeune officier, de rang sénatorial ou équestre, et a une nature purement militaire (cf. par exemple $C I L, X, 7351$, Thermae Himeraeae $=I$. Thermae Himeraeae, 16; X, 4749, Suessa Aurunca). Il suffit de rappeler ici les textes sardes des débuts du $\mathrm{I}^{\mathrm{er}} \mathrm{S}$. apr. J.-C. Quant à l'inscription AIJ, $173=I$. Museum Slovénie, 36, qui mentionne T. Iunius Montanus, sa révision a permis d'éclaircir les problèmes que pose le stemma, et aussi de revenir sur le milieu équestre de la colonie d'Alexandria Troas, îlot de romanité dans un environnement hellénophone.

Le domaine militaire constitue l'un des champs de recherche de la conférence. Plusieurs publications récentes ont ouvert des discussions fructueuses ; ainsi, une inscription de Germisara en Dacie (AMN, 39-41 [2002-2003], 197 et s.) mentionnant un C. Valerius Valentinus, tribun de la légion $X I I I^{a}$ Gemina, puis tribun du numerus singularium Brittannicianorum, en 212-217. On a rappelé l'existence d'homonymes, à ne pas confondre avec cet officier, comme C. Valerius Valentinus (ILBulg., 17, provenance exacte inconnue), primipile et père d'un chevalier romain, connu entre 222 et 
225, ou un autre homonyme, dignitaire municipal à Sarmizegetusa $(C I L$, III, $12590=$ $I D R, I I I / 2,125)$, et insisté sur les dangers que présentaient les identifications indues, dues à des rapprochements purement onomastiques.

Pour les hommes de troupes et simples soldats, le directeur d'études a analysé deux inscriptions grecques d'Olympos en Lycie (Gephyra, 1 [2004], 62, 5, A et B), mentionnant un Marteinus, stationarius, et un Aurelius Mucianus, praetorianus. Ces soldats ont laissé un témoignage de leur piété dans un sanctuaire de la montagne. Des rapprochements ont été faits avec d'autres textes, trois épitaphes venant du même lieu; la première est due d'abord à un certain Aurelius Mucianus, praetorianus, peut-être identifiable avec le précédent (TAM, III, 2, 949); la seconde a été faite pour Aelius Telephus, un Isaurien, bénéficiaire et chef de la statio de l'Olympos (TAM, III, 2, 1165); la troisième, enfin, est l'œuvre du bénéficiaire Iulius Solon. Tous ces témoignages attestent de l'activité militaire déployée dans cette région.

On a aussi traduit en latin l'épitaphe d'un cavalier de la cohors Macedonica, M. Munatius Siluanus, qualifié de heros, dont la lecture (ZPE, 143 [2003], p. 140) a été améliorée par N. Sharankov, ZPE, 148 (2004), p. 198 (AE, 2003, 1599 Neine). On a aussi corrigé le commentaire par $I$. Tyr, 383, de l'épitaphe de C. Autronius C. f. Bassus Fal. Capua, porte-enseigne d'une cohorte Italica milliaria; la mention de l'unité, a donné l'occcasion de présenter les unités auxiliaires cantonnées en Judée, puis en Palestine, en utilisant la documentation issue des diplômes militaires les plus récents. On a fait aussi l'enquête onomastique complète sur les Autronii connus non seulement en Campanie, mais aussi dans le reste de l'Italie.

Depuis des années, les diplômes militaires font l'objet de plusieurs discussions dans le séminaire. Cette année encore, le directeur d'études en a présenté trois : tout d'abord, un texte en provenance d'Aleria, qui vient d'être publié dans Gallia, 62, 2005 (2007), p. 279, mentionnant un marin corse, bénéficiant de la citoyenneté romaine lors de sa mise à la retraite; le préfet de la flotte de Misène à laquelle il appartenait, Iulius Fronto, était déjà connu par ailleurs pour l'année 129, et il faut le distinguer soigneusement d'un anonyme, [-]o, connu aussi comme préfet de la flotte en 119. Deux autres diplômes, encore inédits, ont été signalés, en attendant la publication : l'un en provenance de la Mésie, l'autre venant de la Bétique.

Le reste de la société n'a pas été négligé : ainsi le statut de Valeria Aemil[ina], usu stolae exornata, trium liberorum equitum Romanorum mater (AE, 2003, 1453, Vetus Salina) a été discuté et on a insisté sur la rareté d'une expression n'indiquant pas seulement un privilège accordé à la mère, mais précisant aussi le statut de ses enfants. Des textes, précisant la qualité de pater ou mater equitum Romanorum (duorum uel trium), et utilisés pour l'exaltation d'une famille dans le milieu des collectivités locales, ont été analysés, en portant une attention particulière au contexte local et provincial.

De même, on est revenu sur le mécanisme de l'admissio auprès de l'empereur, avec ses deux degrés, qui rappellent les petites et les grandes entrées de la cour des rois de France, et mis en relief le rôle des affranchis impériaux qui sont chargés de filtrer les personnages venus saluer le prince dès le matin. 